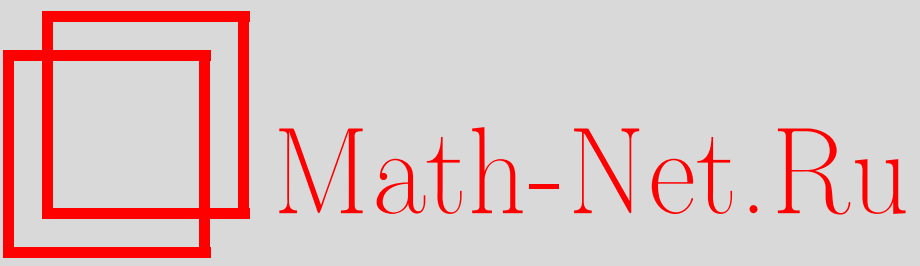

А. В. Павлов, Диффузионные аппроксимации и изменение условий эргодичности при идентичном обслуживании, УМН, 1997, том 52, выпуск 3, 171-172

DOI: https://doi.org/10.4213/rm856

Использование Общероссийского математического портала Math-Net.Ru подразумевает, что вы прочитали и согласны с пользовательским соглашением

http://www.mathnet.ru/rus/agreement

Параметры загрузки:

IP: 54.205 .225 .156

26 апреля 2023 г., $17: 46: 28$ 


\title{
ДИФФУЗИОННЫЕ АППРОКСИМАЦИИ И ИЗМЕНЕНИЕ УСЛОВИЙ ЭРГОДИЧНОСТИ ПРИ ИДЕНТИЧНОМ ОБСЛУЖИВАНИИ
}

\author{
А. В. ПАВЛОВ
}

Рассмотрим $K$ узлов, соединенных последовательно. На $i$-м узле находится $N$ приборов и бесконечное число мест для ожидания, $i=1, \ldots, K$. На первый узел поступает произвольный поток требований, удовлетворяющий единственному ограничению

$$
A_{T}(T) / \lambda(T) T \stackrel{\mathrm{P}}{\rightarrow} 1, \quad 0<C_{1}<\lambda(T)<C_{2} ; \quad C_{1}, C_{2}=\text { const },
$$

где $A_{T}(s)$ - число требований, поступивших на первьй узел за время $s, s \geqslant 0$; все процессы и величины рассматриваются в схеме серий, т.е. зависят от параметра $T \rightarrow \infty$. Обозначим через $\xi_{j}^{i}$ время обслуживания одним любым прибором $i$-го узла требования, поступившего на первый узел $j$-м по счету. В данной статье изучается случай идентичного обслуживания, т.е.

$$
\xi_{j}^{1}=\cdots=\xi_{j}^{K}, \quad j=1,2, \ldots
$$

Следствием идентичности обслуживания являются два интересных факта: во-первых, при сверхвысокой нагрузке на первьй узел $\left(\rho=\lambda \mathrm{M} \xi_{1}^{1}=1\right.$ или $\left.\rho>1\right)$ очередь и время обслуживания на узлах с номерами $2, \ldots, K$ не стремятся к бесконечности; во-вторых, мы имеем пример входного потока требований, у которого при нагрузке $\rho=1$ поведение процесса накопления требований существенно отличается от традиционного поведения обобщенных процессов гибели-размножения, например, при нулевых начальных условиях при $\rho=1$ существует стационарньй режим и очередь ограничена. Предполагается, что процесс $A_{T}(s)$ и последовательность $\left\{\xi_{j}^{1}\right\}$ независимы; случайные величины $\left\{\xi_{j}^{1}\right\}$ независимы и одинаково распределены с функцией распределения $F_{T}(x)=\mathrm{P}\left\{\xi_{1}^{1} \leqslant x\right\}$. В момент окончания обслуживания на $i$-м узле требование мгновенно поступает на $(i+1)$-й узел; на всех узлах требования обслуживаются в порядке поступления. Обозначим символом $Z_{i}^{t}(1)$ суммарное время обслуживания всех требований, находящихся на $i$-м узле в момент времени $t$ (виртуальное время обслуживания для случая $N=1$ ); $Z_{i}^{t}(2)$ - суммарное число требований, находящихся на $i$-м узле в момент времени $t ; Z_{i}^{t}(3)-$ суммарное время пребывания на $i$-м узле требования с номером $n(t)$, где $n(t)$ - номер последнего требования среди всех, поступивших на 1-й узел за время $[0, t] ; i=1, \ldots, K$.

Нам понадобятся условия F1 и F2 $\left(c_{i}\right.$ с произвольным индексом далее обозначают произвольные константы, не зависящие от $T)$.

$\mathrm{F} 1: \sup _{T}\left(1-F_{T}(x)\right)<c_{1} / x^{1+c_{2}}, x \rightarrow \infty, c_{2}>0 ;$

$\mathrm{F} 2$ : для произвольной константы $\beta>1$

$$
\lim _{x \rightarrow \infty} \sup _{T} \bar{F}_{T}(\beta x) / \bar{F}_{T}(x)=0, \quad \bar{F}_{T}(x)=1-F_{T}(x) .
$$

TEOPEMA 1.

$$
\begin{gathered}
\max _{2 \leqslant i \leqslant K} \sup _{0 \leqslant t \leqslant T} \max \left(Z_{i}^{t}(1), Z_{i}^{t}(3)\right) \leqslant(2 N-1) \varkappa(T) ; \\
\varkappa(T)=\max _{1 \leqslant j \leqslant A_{T}(T)} \xi_{j}^{1}, \quad A_{T}(T) \stackrel{\mathrm{P}}{<} \text { const } \cdot T, \quad T \rightarrow \infty .
\end{gathered}
$$

Далее, по определению $\chi(C)$ - индикатор $C$ и

ТЕОРема 2. При выполнении условия F1

$$
\bar{F}_{T}^{(-1)}(x)=\inf x: \bar{F}_{T}(x) \leqslant \delta, \quad 0<\delta<1 .
$$

$$
\mathrm{M} \max _{1 \leqslant j \leqslant n} \xi_{j}^{1} \leqslant c_{*} \bar{F}_{T}^{(-1)}(1 / n), \quad c_{*} \leqslant 2+1 / c_{2} .
$$

При выполнении условия F2 следующее неравенство превращается в равенство, причем $c_{*}=1+o(1), T \rightarrow \infty$. 
Tеорема 3. Существуют такие множества $B(T)$, что $\mathrm{M} Z_{j}^{T}(2) \chi(B(T)) \leqslant \mu(T) \cdot N \cdot(j-1) c_{*} \bar{F}_{T}^{(-1)}(1 / \lambda(T) T) \cdot(1+o(1)), \quad \lim _{T \rightarrow \infty} \mathrm{P}(B(T))=1$, для произвольного $j=2, \ldots, K, \mu(T)=N / \mathrm{M} \xi_{1}^{1}(T)$.

Доказательство теорем 1-3 существенно опирается на равенство для случая $N=1$

$$
Z_{j}^{t}(1)=S_{j}(t)-t-\inf _{0 \leqslant u \leqslant t}\left(S_{j}(u)-u\right), \quad S_{j}(t)=\sum_{i=1}^{A(j, t)} \xi_{i}^{1},
$$

где $A(j, t)$ - число требований, поступивших на $j$-й узел в течение времени $[0, t], j=1, \ldots, K$. Если обозначить через $k(t)$ номер требования, которое обслуживается $(j-1)$-м узлом в момент $t$ (коль скоро такое требование есть), а через $\theta_{j-1}^{t}$ - ту часть длины $\xi_{k(t)}^{j-1}$, которая уже была обслужена к моменту времени $t$ на $(j-1)$-м узле, то

$$
S_{j}(t)=S_{j-1}(t)-\left(Z_{j-1}^{t}(1)+\theta_{j-1}^{t}\right), \quad j=2, \ldots, K .
$$

Подставляя выражение для $Z_{j-1}^{t}(1)$ в данное равенство, получаем (с учетом $\chi\left(Z_{j-1}^{t}(1)=0\right) \times$ $\left.\theta_{j-1}^{t}=0\right)$ :

где $(N=1)$

$$
Z_{j}^{t}(1) \leqslant \theta_{j-1}^{u_{j}(t)}-\theta_{j-1}^{t} \leqslant \varkappa(t), \quad j=2, \ldots, K, \quad u_{j}(t) \leqslant t
$$

$$
\begin{aligned}
u_{j}(t)=\sup \left\{\tau: \inf _{0 \leqslant u \leqslant t}\left[\inf _{0 \leqslant v \leqslant u}\right.\right. & \left.\left(S_{j-1}(v)-v\right)-\theta_{j-1}^{u}\right] \\
& \left.=\inf _{0 \leqslant v \leqslant \tau}\left(S_{j-1}(v)-v\right)-\theta_{j-1}^{\tau}\right\} \cap\{\tau \leqslant t\}, \quad j=2, \ldots, K .
\end{aligned}
$$

Дальнейшее доказательство опирается на методы [2]-[4].

Из теорем 1-3 вытекает

СлЕДСТвиЕ. Если выполнены классические условия диффузионной аппроксимации, то для каждого из $J=1,2,3$ выполнено:

$$
T^{-1 / 2}\left(\sup _{0 \leqslant t \leqslant 1} Z_{2}^{T t}(J), \ldots, \sup _{0 \leqslant t \leqslant 1} Z_{K}^{T t}(J)\right) \stackrel{\mathrm{P}}{\rightarrow}(0, \ldots, 0), \quad J=1,2,3,
$$

причем это утверждение верно, если потребовать только

$$
\sup _{T} \mathrm{M}\left|\xi_{1}^{1}(T)\right|^{2+\varepsilon}<c_{0}<\infty, \quad A_{T}(T)<\lambda_{*} \cdot T, \quad T \rightarrow \infty,
$$

где $\varepsilon>0, c_{0}>0, \lambda_{*}>0-$ произвольные константы.

ЗАмЕчАниЕ. Поскольку приведенные результаты доказьвались без исползования условий эргодичности, так или иначе формулируемых в терминах "нагрузки" меньшей единицы на первьй узел, мы легко получаем пример существенного изменения условий эргодичности на втором узле при любой, в том числе большей единицы, нагрузке на первьй узел.

\section{СПИСОК ЛИТЕРАТУРЫ}

[1] Боровков А. А. Вероятностные процессы в теории массового обслуживания. М.: Наука, 1972. [2] Павлов А. В. // Теория вероятн. и ее примен. 1990. Т. 35. С. 716-726. [3] Павлов А. В. // Теория вероятн. и ее примен. 1991. Т. 36. С. 170-175. [4] Павлов А. В. // УМН. 1996. T. 51. № 1. C. 159-160. 